\title{
Antitumor effects of genetically engineered stem cells expressing yeast cytosine deaminase in lung cancer brain metastases via their tumor-tropic properties
}

\author{
BO-RIM YI ${ }^{1}$, SEUNG U. KIM ${ }^{3,4}$, YUN-BAE KIM ${ }^{2}$, HONG JUN LEE ${ }^{4}$, \\ MYUNG-HAING $\mathrm{CHO}^{5}$ and KYUNG-CHUL $\mathrm{CHOI}^{1}$
}

\begin{abstract}
${ }^{1}$ Laboratory of Veterinary Biochemistry and Immunology and ${ }^{2}$ Laboratory of Toxicology, College of Veterinary Medicine, Chungbuk National University, Cheongju, Chungbuk, Republic of Korea; ${ }^{3}$ Division of Neurology, Department of Medicine, University of British Columbia, Vancouver, British Columbia, Canada; ${ }^{4}$ Medical Research Institute, Chung-Ang University College of Medicine, Seoul; ${ }^{5}$ Laboratory of Toxicology, College of Veterinary Medicine, Seoul National University, Seoul, Republic of Korea
\end{abstract}

Received November 30, 2011; Accepted January 27, 2012

DOI: $10.3892 / o r .2012 .1721$

\begin{abstract}
Although mortality related with primary tumors is approximately $10 \%$, metastasis leads to $90 \%$ of cancerassociated death. The majority of brain metastases result from lung cancer, but the metastatic mechanism remains unclear. In general, chemotherapy for treating brain diseases is disrupted by the brain blood barrier (BBB). As an approach to improve treatment of lung cancer metastasis to the brain, we employed genetically engineered stem cells (GESTECs), consisting of neural stem cells (NSCs) expressing a suicide gene. Cytosine deaminase (CD), one of the suicide genes, originating from bacterial (bCD) or yeast (yCD), which can convert the nontoxic prodrug, 5-fluorocytosine (5-FC), into 5-fluorouracil (5-FU), can inhibit cancer cell growth. We examined the therapeutic efficacy and migratory properties of GESTECs expressing yCD, designated as HB1.F3.yCD, in a xenograft mouse model of lung cancer metastasis to the brain. In this model, A549 lung cancer cells were implanted in the right hemisphere of the mouse brain, while CM-DiI pre-stained HB1.F3.yCD cells were implanted in the contralateral brain. Two days after the injection of stem cells, 5-FC was administered via intraperitoneal injection. The tumor-tropic effect of HB1.F3.yCD was evident by fluorescent analysis, in which red-colored stem cells migrated to the lung tumor mass of the contralateral brain. By histological analysis of extracted brain,
\end{abstract}

Correspondence to: Dr Kyung-Chul Choi, Laboratory of Veterinary Biochemistry and Immunology, College of Veterinary Medicine, Chungbuk National University, Cheongju, Chungbuk 361-763, Republic of Korea

E-mail:kchoi@cbu.ac.kr

Key words: lung cancer, tumor tropism, stem cells, cytosine deaminase, brain metastasis the therapeutic efficacy of HB1.F3.yCD in the presence of 5-FC was confirmed by the reduction in density and aggressive tendency of lung cancer cells following treatment with 5-FC, compared to a negative control or HB1.F3.yCD injection without 5-FC. Taken together, these results indicate that HB1. F3.yCD expressing a suicide gene may be a new therapeutic strategy for lung cancer metastases to the brain in the presence of a prodrug.

\section{Introduction}

Metastasis is an insidious movement of cancer cells from primary tumor sites to distant organs and tissues, including the brain, liver, and bones, via blood and lymphatic vessels. Metastasis accounts for over $90 \%$ of lethality in cancer patients (1). Particularly, brain metastasis is the most common intracranial neoplasm and arises in $10-40 \%$ of all cancer patients (2). Because metastases in the brain may rapidly compromise central nervous system (CNS) function, it is a significant cause of cancer-related morbidity and mortality worldwide (3). The most common origins of brain metastases include primary cancers of the lung, breast and skin (4). Lung cancer, the most prevalent cancer in men, is the leading cause of cancer-related death in the developed world (5). Of all malignancies, primary lung cancer has the highest incidence for brain metastasis and approximately $40 \%$ of all patients with lung cancer develop brain metastasis, followed by breast cancer (6). For treating these brain metastases, therapies usually include surgery, chemotherapy, and radiotherapy, but these therapies have many side effects in the nervous system (7). Therefore, brain metastasis is a very critical problem in the overall management of lung cancer.

In this study, seeking a treatment for lung cancer metastasis to the brain, neural stem cells (NSCs) derived from human fetal telencephalon were used for a genetically engineered stem cell (GESTEC)-based therapy (8). GESTECs were engineered to include the gene for a direct prodrug/enzyme (GEPT) system producing bioactive enzymes that convert a relatively 


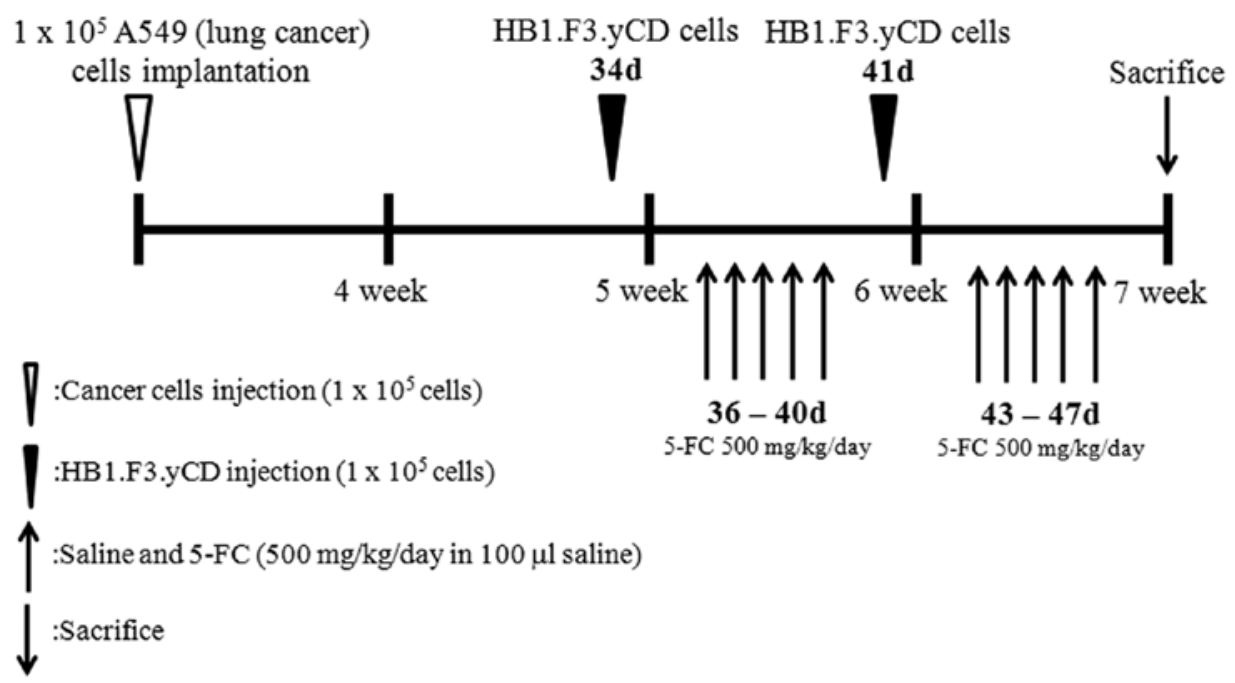

Figure 1. In vivo experimental plan. Human non-small cell lung adenocarcinoma A549 cells $\left(1 \times 10^{5}\right.$ cells in $10 \mu 1$ saline $)$ were implanted into the brain of 7-week-old SCID mice (n=15). After 34 to 41 days of implantation, HB1.F3.yCD cells (1x10 cells in $10 \mu 1$ saline) and 5-FC (500 mg/kg/day in $100 \mu 1 \mathrm{saline})$ were introduced via contralateral and intraperitoneal injection, respectively. The mice were divided into three groups. In group 1, the mice were treated with 10 or $100 \mu 1$ saline as a negative control. In group 2, the mice were treated with HB1.F3.yCD cells and $100 \mu 1$ saline in the absence of 5-FC. In group 3, the mice were treated with HB1.F3.yCD and 5-FC ( $500 \mathrm{mg} / \mathrm{kg} / \mathrm{day}$ in $100 \mu \mathrm{l}$ saline). At 2 days following the last 5-FC injection, all of the mice treated with lung cancer cells were euthanized.

non-toxic prodrug into a toxic agent (9). Cytosine deaminase (CD)/5-fluorocytosine (5-FC), one of the most widely used GEPT systems, is expressed in bacteria like Escherichia coli or yeast like Saccharomyes cerevisiae. In both organisms, the encoded CD catalyzes the conversion of a 5-FC prodrug into a toxic agent, 5-fluorouracil (5-FU), which is able to inhibit cancer cell growth $(10,11)$ by impairing DNA synthesis and promoting apoptosis (12). However, yeast CD (yCD) appears to be far more efficient in the conversion of 5-FC to 5-FU than bacterial CD (bCD) in both in vitro and in vivo models (13). In addition, a variety of stem cells as well as NSCs exert tumor tropic effects $(14,15)$. Thus, we previously produced yCD-transfected GESTECs, designated as HB1.F3.yCD, as a superior delivery system to treat metastatic lung cancer to brain or other tissues.

In this study, we employed an immortalized HB1.F3.yCD to selectively target metastatic lung cancer to the brain. The metastatic breast cancer to the brain was proved by using HB1.F3.bCD in the previous study (16). We confirmed the therapeutic efficacy and migratory property of HB1.F3.yCD for lung cancer metastasis to the brain in vivo. Overall, these results suggest that GESTECs expressing a therapeutic gene (yCD) can be an excellent therapeutic system to effectively target metastatic lung cancer to the brain or other organs.

\section{Materials and methods}

Cell culture. The A549 human non-small cell lung adenocarcinoma cell line was purchased from the Korean Cell Line Bank (KCLB, Seoul, Korea). These cells were cultured in containing Dulbecco's modified Eagle's Medium (DMEM; Hyclone Laboratories, Logan, UT, USA) containing $10 \%$ (v/v) heat-inactivated fetal bovine serum (FBS; Hyclone Laboratories), $1 \%$ penicillin and $1 \%$ streptomycin (Cellgro Mediatech, Manassas, VA, USA), 1\% HEPES (Invitrogen Life
Technologies, Carlsbad, CA, USA), and plasmocin (InvivoGen, San Diego, CA, USA). HB1.F3.yCD engineered stem cells were cultured in the same medium.

5-FC and 5-FU effects on lung cancer cell growth. To confirm

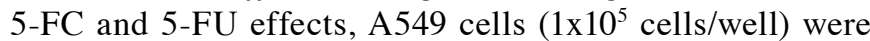
seeded in 6-well plates containing the aforementioned medium modified to contain 5\% FBS. Twenty-four hours after cancer cell incubation, 5-FC (0.5 mmol/1; Sigma-Aldrich, St. Louis, MO, USA) and 5-FU (0.5 mmol/l; Sigma-Aldrich) were added to each well for 4 days. These results were observed by inverted microscopy using an IX71 microscope (Olympus, Tokyo, Japan). Next, to ascertain the effects of various concentrations of 5-FU (0.005-1.0 mmol/1) on lung cancer cells, $5 \times 10^{3}$ A549 cells were cultured in a 96-well plate for 1 day. 5-FU of different concentrations was added to each well and incubation was continued for 4 days. A cell viability assay was conducted. Briefly, $10 \mu \mathrm{l}$ of $5 \mathrm{mg} / \mathrm{ml} \mathrm{3-(4-,5-dimethylthiazol-}$ 2-yl)-2,5-diphenyl tetrazolium bromide (MTT) stock solution dissolved in phosphate-buffered saline (PBS) was added to each well and the plate was incubated for $4 \mathrm{~h}$ in darkness at $37^{\circ} \mathrm{C}$. Insoluble formazan crystals that formed were dissolved in $100 \mu \mathrm{l}$ dimethyl sulfoxide (DMSO; Junsei Chemical, Co., Ltd., Tokyo, Japan) and the absorbance was measured at $540 \mathrm{~nm}$ using a VersaMax ELISA microplate reader (Molecular Devices, Sunnyvale, CA, USA). Each experiment was conducted in duplicate $(n=6)$.

In vitro therapeutic efficacy of HB1.F3.yCD. To explain the therapeutic efficacy of HB1.F3.yCD, A549 cells $\left(4 \times 10^{3}\right)$ were cultured in a 96 -well plate and incubated at $37^{\circ} \mathrm{C}$ for one day. Twenty-four hours after incubation HB1.F3.yCD cells $\left(8 \times 10^{3}\right)$ were co-cultured with A549. A day later, 5-FC diluted in saline $(100,200,300,400$, and $500 \mu \mathrm{g} / \mathrm{ml})$ was added in culture wells and incubation continued for 4 days. To confirm cell viability, 


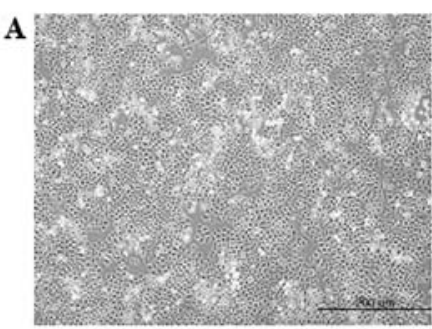

5-FC

B

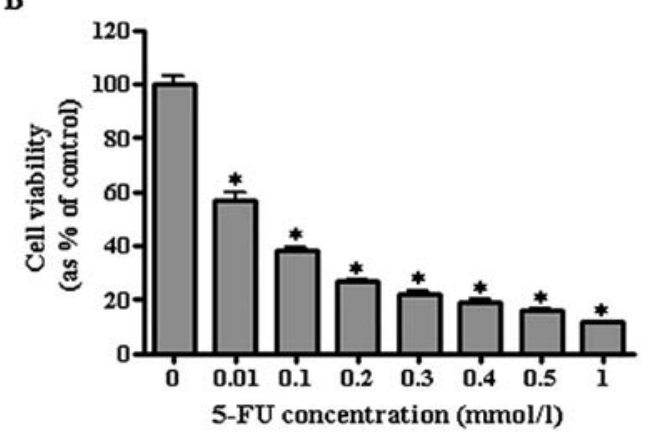

Figure 2. Effects of 5-FC and 5-FC on the cell viability of human lung cancer cells. (A) A549 lung cancer cells (A549, $1 \times 10^{5}$ cells per well) were seeded in 6-well plates for $24 \mathrm{~h}$. After seeding, 5-FC and 5-FU (0.5 mmol/l) were treated in lung cancer cells cultured for 4 days. Each well was observed by microscopy. Magnification, $x 100$. (B) A549 lung cancer cells $\left(5 \times 10^{3}\right.$ cells per well) were seeded in 96-well plates for $24 \mathrm{~h}$. Increasing concentrations of 5 -FU at $0.01,0.1,0.2,0.3,0.4,0.5$, and $1.0 \mathrm{mmol} / 1$ were treated in cultured 96-well plate for 4 days. After 4 days, the MTT assay was performed to determine cell viability. ${ }^{*} \mathrm{P}<0.05$ vs. a negative control.

MTT solution was added in each well for $4 \mathrm{~h}$ and absorbance was measured at $540 \mathrm{~nm}$. The experiments were performed in duplicate.

Animal model of brain metastasis. All animal experiments were approved by the Animal Care Committee of Chungbuk National University. All animals were acclimated at $24-26^{\circ} \mathrm{C}$ and $40-60 \%$ relative humidity under a $12 \mathrm{~h}$ light/dark cycle and frequent ventilation. Fifteen male SCID mice (7-week-old) were obtained from the Korea Research Institute of Bioscience and Biotechnology (KRIBB, Ochang, Korea). After being anesthetized, SCID mice each received A549 (1x10 5 cells in $10 \mu \mathrm{l}$ saline) via injection into white matter at a depth of $2 \mathrm{~mm}$ [anterior/posterior (AP) $+1.0 \mathrm{~mm}$, medial/lateral (ML) $+1.7 \mathrm{~mm}$, dorsal/ventral (DV) $-3.2 \mathrm{~mm}$ ].

In vivo therapeutic efficacy of HB1.F3.yCD. Four weeks after implantation, saline $(10 \mu \mathrm{l})$ or HB1.F3.yCD $\left(1 \times 10^{5}\right.$ cells in $10 \mu \mathrm{l}$ saline) was introduced via contralateral injection (AP +1.0 mm, ML -1.7 mm, DV -3.2 mm). HB1.F3.yCD cells were preincubated with CellTracker ${ }^{\mathrm{TM}} \mathrm{CM}-\mathrm{DiI}$ (Invitrogen Life Technologies). Briefly, stem cells were treated with $5 \mu \mathrm{M}$ CM-DiI for $5 \mathrm{~min}$ at $37^{\circ} \mathrm{C}$ and for $15 \mathrm{~min}$ at $4^{\circ} \mathrm{C}$. After two washes in PBS, the defined number of stem cells was injected into the brain. One hundred microliters or $500 \mathrm{mg} / \mathrm{kg} /$ day $5-\mathrm{FC}$ in $100 \mu \mathrm{l}$ was administered via intraperitoneal injection. The total number of mice were divided into three groups $(n=5$ per group). Group 1 was treated with $10 \mu \mathrm{l}$ saline as a negative control. Group 2 was treated with HB1.F3.yCD in the absence of 5-FC in $100 \mu \mathrm{l}$ saline. Group 3 was treated with HB1. F3.yCD in the presence of $5-\mathrm{FC}(500 \mathrm{mg} / \mathrm{kg} /$ day $)$ in $100 \mu \mathrm{l}$ saline. Two days following the last injection with 5-FC, all mice were euthanized and brains were collected. The in vivo experimental scheme using a xenograft model is shown in Fig. 1.

Histopathological and fluorescence analysis. The extracted brain was sliced in 4-6 mm-thick sections and fixed in $10 \%$ normal formalin solution (Sigma-Aldrich), embedded in paraffin, and sectioned at a thickness of $5 \mu \mathrm{m}$ using a microtome. Tissues were stained using hematoxylin and eosin following the general protocol and observed by microscopy using a model BX51 microscope (Olympus).

To confirm tumor-tropism effects of stem cell via fluorescence analysis, CM-DiI pre-stained HB1.F3.yCD was injected in mouse brain. After brain sections were obtained as described above, each slide was treated with $10 \%$ normal formalin solution for $10 \mathrm{~min}$ and washed with PBS two times. 4',6-Diamidino-2-phenylindole (DAPI) solution was added dropwise to each slide and incubated for $10 \mathrm{~min}$ at $37^{\circ} \mathrm{C}$ in darkness. Stained slides were observed using an IX71 inverted microscope (Olympus).

Statistical analysis. Data of each experiment are shown as the mean \pm standard deviation (SD). To evaluate the significance of each in vitro group, statistical analysis was performed by the one-way ANOVA Tukey's test using the GraphPad Prism software (v5.0; GraphPad Software, San Diego, CA, USA). $\mathrm{P}<0.05$ were considered to indicate statistically significant differences.

\section{Results}

Cytotoxic effects of 5-FC and 5-FU for lung cancer cells. In the 6-well plate culture system, we confirmed the effects of 5-FC and 5-FU on the viability of lung cancer cells. When A549 cells were treated with $0.5 \mathrm{mmol} / \mathrm{l}$ of 5-FC, there was no change in the cell growth (Fig. 2A). However, treatment with $0.5 \mathrm{mmol} / \mathrm{l} 5-\mathrm{FU}$ resulted in significant inhibition of A549 lung cancer cell growth as shown in Fig. 2A. To confirm the effects of 5-FU at various concentrations $(0.01,0.1,0.2,0.3$, $0.4,0.5$ and $1.0 \mathrm{mmol} / \mathrm{l})$, the cell viability was measured by an MTT assay following culture of A549 cells in 96-well plates. Inhibition of lung cancer cell growth was observed in a dosedependent manner and the cell viability was suppressed up to approximately $80 \%$ as seen in Fig. 2B.

Therapeutic efficacy of HB1.F3.yCD and 5-FC. Based on the effect of 5-FU on A549 cell growth, we employed a co-culture system in which cancer and stem cells were seeded in the same wells, and cell viability was measured by the MTT assay. In the presence of 5-FC and HB1.F3.yCD, the viability of A549 lung cancer cells was reduced $20-40 \%$, and the maximal inhibition was observed at $500 \mu \mathrm{g} / \mathrm{ml}$ of $5-\mathrm{FC}$ in the presence of HB1.F3.yCD (Fig. 3). The growth of A549 lung cancer cells was not altered following treatment with a maximal dose of 5-FC $(500 \mu \mathrm{g} / \mathrm{ml})$ in the absence of HB1.F3.yCD (data not shown). 


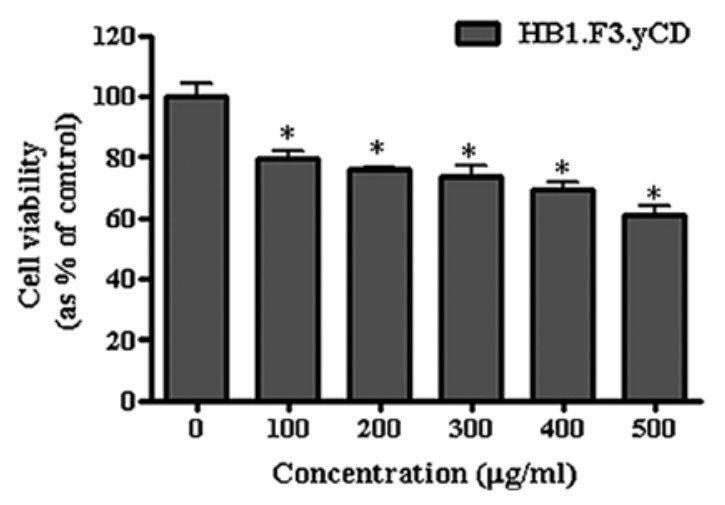

Figure 3. In vitro therapeutic efficacy of HB1.F3.yCD cells. A549 lung cancer cells $\left(4 \times 10^{3}\right.$ cells per well) were cultured in 96-well plates for one day. Following implantation of HB1.F3.yCD cells $\left(8 \times 10^{3}\right.$ cells per well), increasing concentrations of 5-FC at $0,100,200,300,400$ and $500 \mu \mathrm{g} / \mathrm{ml}$ were added into each well for 4 days. Cell viability was measured by the MTT assay. ${ }^{*} \mathrm{P}<0.05$ vs. control $(0 \mu \mathrm{g} / \mathrm{ml})$.

In vivo migratory capacity of HB1.F3.yCD cells. We confirmed the in vivo migratory property of HB1.F3.yCD cells toward lung metastatic cancer to the brain. Lung metastases to the brain were mimicked by implantation of A549 cells into the right hemisphere and CM-DiI pre-stained HB1.F3.yCD cells into the left hemisphere. DAPI counterstaining was performed and the migration of CM-DiI-labeled stem cells was traced via microscopy. By fluorescence analysis, red-colored HB1. F3.yCD cells were observed in the contralateral brain as shown in Fig. 4, indicating that injected HB1.F3.yCD cells have a migratory property towards A549 lung cancer cells injected into brain in vivo.
In vivo therapeutic efficacy of HB1.F3.yCD cells. To confirm the therapeutic effect of HB1.F3.yCD cells in the mouse brain, a xenograft model was employed as previously described $(9,16)$. At 2 days following the last 5-FC injection, the brains of lung cancer cell-bearing SCID mice were extracted and examined by histological and fluorescence analyses. The implanted lung tumor mass in the brain was not reduced in the control mice in the presence of HB1.F3.yCD without 5-FC or in the absence of HB1.F3.yCD with 5-FC. In contrast, a significant reduction of lung tumor mass was observed in the brain of SCID mice treated with HB1.F3.yCD in the presence of 5 -FC ( $500 \mathrm{mg} / \mathrm{kg} /$ day $)$ as demonstrated in Fig. 5 . The implanted lung cancer cells and normal mouse brain cells were distinguished, and the nuclear to cytoplasmic ratio was increased in lung cancer cells compared to normal brain cells observed by histological analysis. In addition, the density of lung cancer cells in the formed tumor masses was significantly decreased by HB1.F3.yCD expressing yCD in the presence of 5-FC (Fig. 5).

\section{Discussion}

Brain metastases are the most common malignant tumor of the CNS outnumbering primary brain tumors such as glioma and glioblastoma (17). Although the major requirements for metastasis to distant sites remain incompletely understood, clinically, brain metastases most commonly arise from lung, breast and skin cancers $(18,19)$. Such factors have not yet been identified for brain metastasis and the most common treatment approaches for brain metastasis are surgery, radiotherapy, chemotherapy and stereotactic radiotherapy (20). However, these therapies carry the risk of development of neurological and cognitive deficits and, in the case of chemotherapy, the
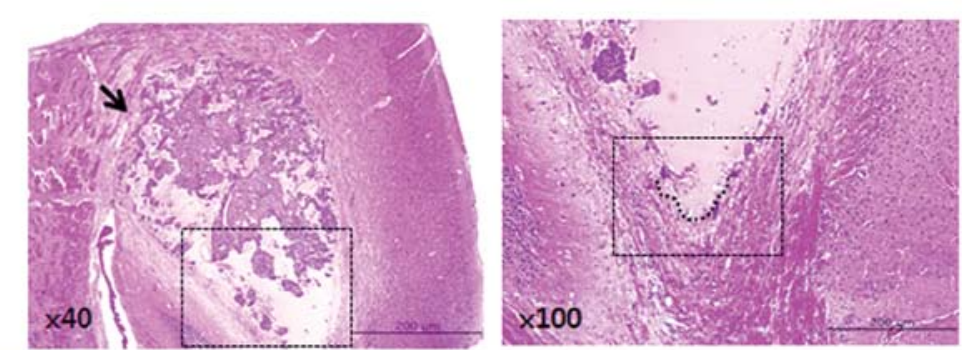

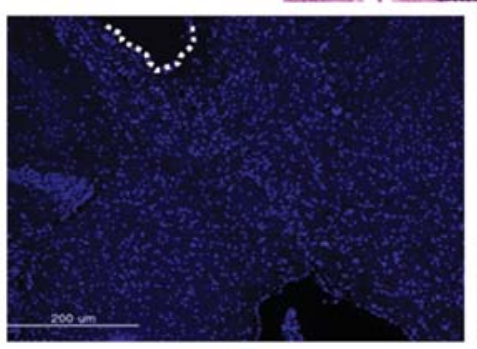

DAPI

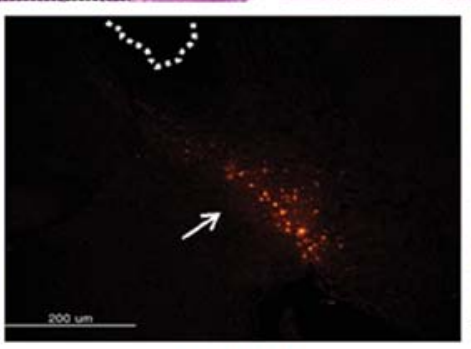

CM-DiI

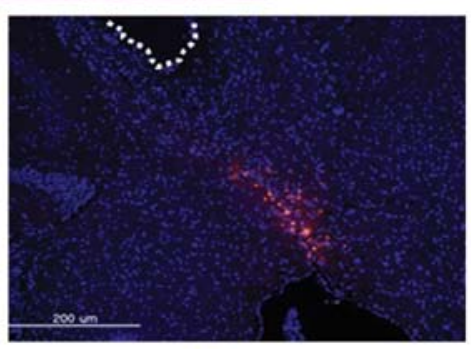

Merge

Figure 4. In vivo migratory ability of stem cells towards lung cancer metastasis in the brain. The upper panels show brain tissue treated with HB1.F3.yCD and 5-FC by hematoxylin and eosin staining. The lower panels present the brain tissues following injection with A549 lung cancer cells and HB1.F3.yCD by fluorescence analysis. To observe migratory effects, brain sections were stained by DAPI for fluorescence analysis. Red, pre-stained HB1.F3.yCD; blue, stained brain and HB1.F3.yCD cells; black and white dotted line, the boundary of tumor and normal brain cells; black arrow, tumor mass by H\&E staining; white arrow, migrated stem cells. 

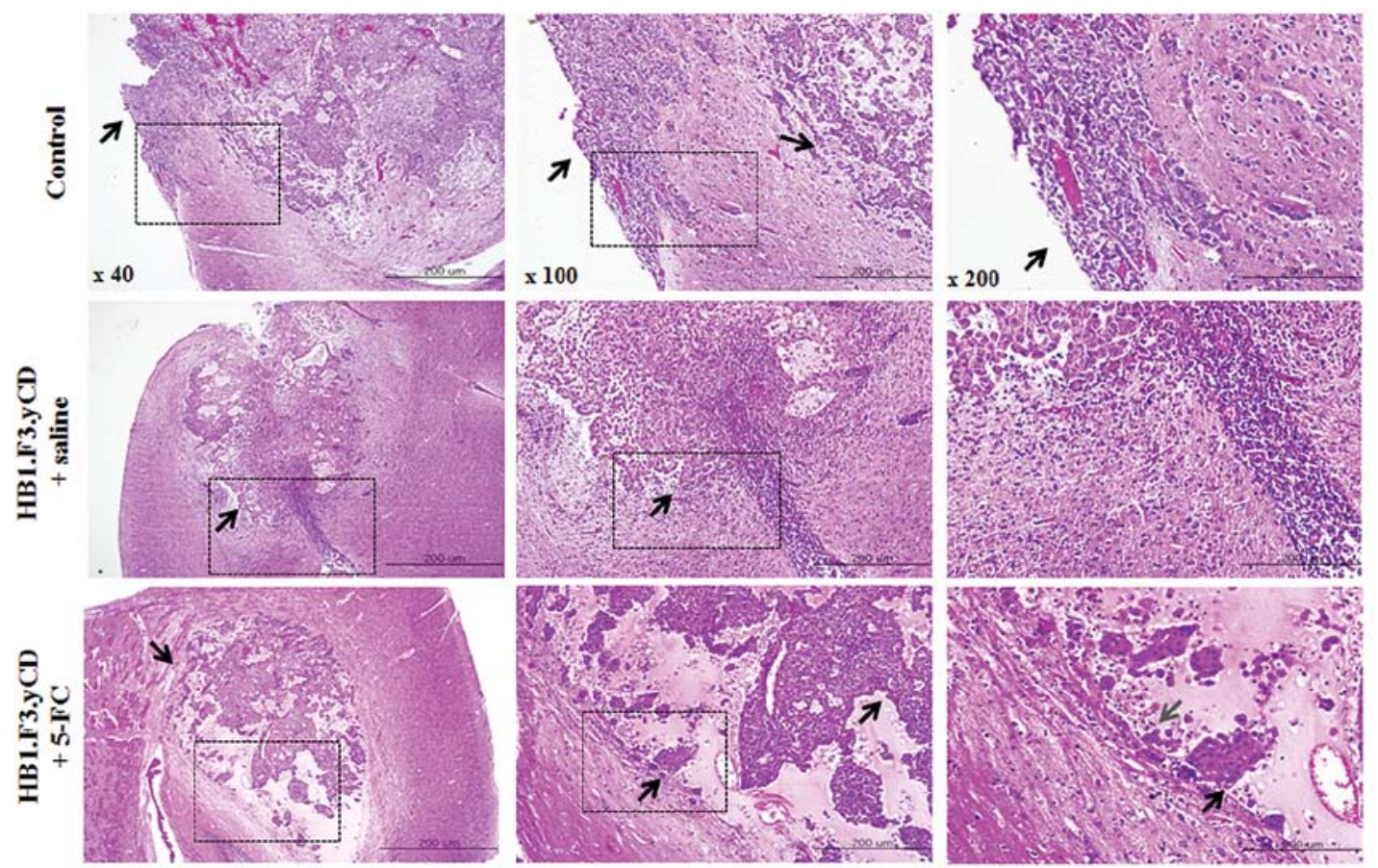

Figure 5. In vivo therapeutic efficacy of HB1.F3.yCD cells. After tumor formation in the right hemisphere of the mouse brain, HB1.F3.yCD cells pre-stained with CM-DiI were implanted into the contralateral region of the brain. After 2 days of injection, $5-\mathrm{FC}$ (500 mg/kg/day) was intraperitoneally injected into the SCID mice. The extracted brains were analyzed for tumor formation by HB1.F3.yCD cells in the presence of a prodrug, 5-FC. The dotted rectangle areas are enlarged in the images to the right by microscopy. Black arrow, injected A549 lung cancer cells; gray arrow, dying lung cancer cells.

blood-brain barrier (BBB) is disrupted, enhancing brain metastases $(21,22)$.

Stem cells have been proposed as the basis of alternative therapy. In particular, NSCs can differentiate to neural cell lineages, including astrocytes and oligodendrocytes, in vitro and in vivo (23). Immortalized NSCs expressing a selected suicide gene can inhibit tumor growth in prostate, lung, and endometrial cancer, as well as brain diseases $(9,16)$. In addition, NSCs have a tumor-tropic effect that includes their migration toward tumor cells (24). A GESTECs based GEPT system, including $\mathrm{CD} / 5-\mathrm{FC}$ and carboxyl esterase (CE)/ irinotecan (CPT-11), can effetely deliver therapeutic genes and decrease growth of tumor cells in vitro and in vivo $(25,26)$. The CD/5-FC system, a GEPT system, is used for bCD and yCD genes, which convert non-toxic prodrug 5-FC into toxic 5-FU. 5-FU inhibits RNA processing and DNA synthesis in cancer cells and ultimately results in cell death (27). Therefore, stem cells, particularly NSCs, may be an excellent vehicle as a delivery system of therapeutic genes.

In this study, we confirmed the therapeutic efficacy and migratory ability of HB1.F3.yCD cells expressing the $\mathrm{yCD}$ gene in in vitro and in vivo models. Presently, lung cancer cells were very sensitive to even low concentrations $(0.01 \mathrm{mmol} / \mathrm{l})$ of 5-FU, but 5-FC was not effective in the growth of lung cancer cells. HB1.F3.yCD cells showed a $40 \%$ inhibition of cell viability for lung cancer cells in the presence of 5-FC $(500 \mu \mathrm{g} / \mathrm{ml})$ and their therapeutic efficacy was confirmed in the in vitro model.

Using a mouse model of lung cancer cell metastasis to the brain, we also verified the effect of HB1.F3.yCD cells in brain metastasis by tumor tropism in vivo. After implantation of lung cancer cells into the right hemisphere, CM-DiI-pre-stained HB1.F3.yCD cells were injected into the contralateral brain of SCID mice following injection with A549 lung cancer cells. The red-colored HB1.F3.yCD cells appeared to migrate to the area of lung metastasis in the brain, as observed by fluorescence analysis. This migratory property of HB1.F3.yCD cells can be caused by various chemoattractant factors secreted by cancer cells including stromal cell-derived factor-1, vascular endothelial growth factor, and monocyte chemoattractant protein-1 (28). Secreted ligands may be recognized by HB1.F3.yCD cells, and a tumor specific migratory property may be stimulated by these stem cells (29).

Among them, the use of HB1.F3.yCD cells in the presence of 5-FC showed that the generated tumor mass of lung tumor metastasis was significantly reduced compared to a negative control or HB1.F3.yCD in the absence of 5-FC. In histological analysis, we further confirmed the inhibitory effect of HB1.F3.yCD cells on lung tumor formation in the mouse brain. In particular, the number of lung tumor cells was evidently reduced, and the aggressive character of the tumor cells was decreased by the injection of HB1.F3.yCD in the presence of 5-FC. Taken together, these results indicate that lung cancer metastasis to the brain can be cured by HB1.F3.yCD cells in the conversion of a prodrug, 5-FC, to its metabolic active form, 5-FU, via their tumor tropic effect.

In summary, the viability of lung cancer cells was inhibited by co-culturing with HB1.F3.yCD in the presence to 5-FC in vitro. In addition, the therapeutic efficacy and migratory ability of HB1.F3.yCD cells were verified in a mouse xenograft model in vivo. Although pre-stained HB1.F3.yCD cells were injected into the contralateral area of the brain following 
formation of lung cancer, they can migrate to the tumor mass of lung cancer cells and kill them via tumor tropism. These results suggest that HB1.F3.yCD cells have tumor-tropic and tumoricidal effects in the presence of a prodrug, 5-FC, suggesting that these GESTECs may be an effective therapeutic strategy for lung cancer metastasis to brain.

\section{Acknowledgements}

This study was supported by a National Research Foundation of Korea (NRF) grant funded by the Ministry of Education, Science and Technology (MEST) of the Korean government (no. 2010-0003093). In addition, this study was also supported by the Priority Research Centers Program through the NRF funded by the MEST (2009-0094035).

\section{References}

1. Li F, Tiede B, Massague J and Kang Y: Beyond tumorigenesis: cancer stem cells in metastasis. Cell Res 17: 3-14, 2007.

2. Gavrilovic IT and Posner JB: Brain metastases: epidemiology and pathophysiology. J Neurooncol 75: 5-14, 2005.

3. Barnholtz-Sloan JS, Sloan AE, Davis FG, Vigneau FD, Lai P and Sawaya RE: Incidence proportions of brain metastases in patients diagnosed (1973 to 2001) in the Metropolitan Detroit Cancer Surveillance System. J Clin Oncol 22: 2865-2872, 2004.

4. Ma S, Xu Y, Deng Q and Yu X: Treatment of brain metastasis from non-small cell lung cancer with whole brain radiotherapy and Gefitinib in a Chinese population. Lung Cancer 65: 198-203, 2009.

5. Poulsen TT, Pedersen N and Poulsen HS: Replacement and suicide gene therapy for targeted treatment of lung cancer. Clin Lung Cancer 6: 227-236, 2005.

6. Schuette W: Treatment of brain metastases from lung cancer: chemotherapy. Lung Cancer 45 (Suppl 2): S253-S257, 2004.

7. Kim SU: Neural stem cell-based gene therapy for brain tumors. Stem Cell Rev 7: 130-140, 2010.

8. Kim KY, Kim SU, Leung PC, Jeung EB and Choi KC: Influence of the prodrugs 5-fluorocytosine and CPT-11 on ovarian cancer cells using genetically engineered stem cells: tumor-tropic potential and inhibition of ovarian cancer cell growth. Cancer Sci 101: 955-962, 2010

9. Yi BR, Kang NH, Hwang KA, Kim SU, Jeung EB and Choi KC: Antitumor therapeutic effects of cytosine deaminase and interferon- $\beta$ against endometrial cancer cells using genetically engineered stem cells in vitro. Anticancer Res 31: 2853-2861, 2011.

10. Ramnaraine M, Pan W, Goblirsch M, Lynch C, Lewis V, Orchard P, Mantyh P and Clohisy DR: Direct and bystander killing of sarcomas by novel cytosine deaminase fusion gene. Cancer Res 63: 6847-6854, 2003.

11. Van Rite BD and Harrison RG: Annexin V-targeted enzyme prodrug therapy using cytosine deaminase in combination with 5-fluorocytosine. Cancer Lett 307: 53-61, 2011.

12. Chen JK, Hu LJ, Wang D, Lamborn KR and Deen DF: Cytosine deaminase/5-fluorocytosine exposure induces bystander and radiosensitization effects in hypoxic glioblastoma cells in vitro. Int J Radiat Oncol Biol Phys 67: 1538-1547, 2007.

13. Nyati MK, Symon Z, Kievit E, Dornfeld KJ, Rynkiewicz SD, Ross BD, Rehemtulla A and Lawrence TS: The potential of 5-fluorocytosine/cytosine deaminase enzyme prodrug gene therapy in an intrahepatic colon cancer model. Gene Ther 9: 844-849, 2002.
14. Tang Y, Shah K, Messerli SM, Snyder E, Breakefield X and Weissleder R: In vivo tracking of neural progenitor cell migration to glioblastomas. Hum Gene Ther 14: 1247-1254, 2003.

15. Lee DH, Ahn Y, Kim SU, Wang KC, Cho BK, Phi JH, Park IH, Black PM, Carroll RS, Lee J and Kim SK: Targeting rat brainstem glioma using human neural stem cells and human mesenchymal stem cells. Clin Cancer Res 15: 4925-4934, 2009.

16. Yi BR, O SN, Kang NH, Hwang KA, Kim SU, Jeung EB, Kim YB, Heo GJ and Choi KC: Genetically engineered stem cells expressing cytosine deaminase and interferon- $\beta$ migrate to human lung cancer cells and have potentially therapeutic antitumor effects. Int J Oncol 39: 833-839, 2011.

17. Carbonell WS, Ansorge O, Sibson N and Muschel R: The vascular basement membrane as 'soil' in brain metastasis. PLoS One 4: e5857, 2009.

18. Yoneda T, Williams PJ, Hiraga T, Niewolna M and Nishimura R: A bone-seeking clone exhibits different biological properties from the MDA-MB-231 parental human breast cancer cells and a brain-seeking clone in vivo and in vitro. J Bone Miner Res 16: 1486-1495, 2001.

19. Sheridan C, Kishimoto H, Fuchs RK, Mehrotra S, Bhat-Nakshatri P, Turner $\mathrm{CH}$, Goulet $\mathrm{R}$ Jr, Badve $\mathrm{S}$ and Nakshatri H: CD44 ${ }^{+} / \mathrm{CD} 24^{-}$breast cancer cells exhibit enhanced invasive properties: an early step necessary for metastasis. Breast Cancer Res 8: R59, 2006.

20. Nguyen DX and Massague J: Genetic determinants of cancer metastasis. Nat Rev Genet 8: 341-352, 2007.

21. van den Bent MJ: The role of chemotherapy in brain metastases. Eur J Cancer 39: 2114-2120, 2003.

22. Joo KM, Park IH, Shin JY, Jin J, Kang BG, Kim MH, Lee SJ, Jo MY, Kim SU and Nam DH: Human neural stem cells can target and deliver therapeutic genes to breast cancer brain metastases. Mol Ther 17: 570-575, 2009.

23. Hanke P, Serwe M, Dombrowski F, Sauerbruch T and Caselmann WH: DNA vaccination with AFP-encoding plasmid DNA prevents growth of subcutaneous AFP-expressing tumors and does not interfere with liver regeneration in mice. Cancer Gene Ther 9: 346-355, 2002

24. Kim SU: Human neural stem cells genetically modified for brain repair in neurological disorders. Neuropathology 24: 159-171, 2004.

25. Gutova M, Najbauer J, Chen MY, Potter PM, Kim SU and Aboody KS: Therapeutic targeting of melanoma cells using neural stem cells expressing carboxylesterase, a CPT-11 activating enzyme. Curr Stem Cell Res Ther 5: 273-276, 2010.

26. Seol HJ, Jin J, Seong DH, Joo KM, Kang W, Yang H, Kim J, Shin CS, Kim Y, Kim KH, et al: Genetically engineered human neural stem cells with rabbit carboxyl esterase can target brain metastasis from breast cancer. Cancer Lett 311: 152-159, 2011.

27. Bencharit S, Morton CL, Howard-Williams EL, Danks MK, Potter PM and Redinbo MR: Structural insights into CPT-11 activation by mammalian carboxylesterases. Nat Struct Biol 9: 337-342, 2002.

28. Aboody KS, Najbauer J and Danks MK: Stem and progenitor cell-mediated tumor selective gene therapy. Gene Ther 15: 739-752, 2008.

29. Gutova M, Najbauer J, Frank RT, Kendall SE, Gevorgyan A, Metz MZ, Guevorkian M, Edmiston M, Zhao D, Glackin CA, et al: Urokinase plasminogen activator and urokinase plasminogen activator receptor mediate human stem cell tropism to malignant solid tumors. Stem Cells 26: 1406-1413, 2008. 